\title{
Sponge-like Porous ZnO Photoanodes for Highly Efficient dye-sensitized Solar Cells
}

\author{
A. $\mathrm{SACCO}^{a}$, A. Lamberti ${ }^{a, b}$, I. Berardone ${ }^{b}, \mathrm{~S} \mathrm{Bianco}^{a, *}, \mathrm{R} . \mathrm{GaziA}^{a}, \mathrm{D} . \mathrm{Pugliese}^{a, b}$, \\ M. QUAGLio ${ }^{a}$, E. TResso ${ }^{a, b}$ AND C.F. Pirri ${ }^{a, b}$ \\ ${ }^{a}$ Center for Space Human Robotics @ PoliTo, Istituto Italiano di Tecnologia, Corso Trento 21, Torino, 10129, Italy \\ ${ }^{b}$ Dipartimento Scienza Applicata e Tecnologia, Politecnico di Torino \\ Corso Duca degli Abruzzi 24, Torino, 10129, Italy
}

\begin{abstract}
We propose a 3D branched $\mathrm{ZnO}$ nanostructure for the fabrication of highly efficient dye-sensitized solar cell photoanodes. A coral-shaped structured Zn layer was deposited by radio frequency magnetron sputtering at room temperature onto fluorine-doped tin oxide/glass sheets and then thermally oxidized in ambient atmosphere, obtaining a high-density branched $\mathrm{ZnO}$ film. The porous structure provides a large surface area, and, as a consequence, a high number of adsorption sites, and the size and spacing of the nanostructures (on the order of the exciton diffusion length) are optimal for good electron collection efficiency. The proposed synthesis technique is simple and scalable and the reproducibility of the growth results was tested. The crystalline phase of the film was investigated, evidencing the complete oxidation and the formation of a pure wurtzite crystalline structure. ZnO-based solar harvesters were fabricated in a microfluidic architecture, using conventional sensitizer and electrolyte. The dependence of the cell efficiency on dye incubation time and film thickness was studied with $I-V$ electrical characterization and electrochemical impedance spectroscopy. The obtained conversion efficiency values, with a maximum value of $4.83 \%$, confirm the highly promising properties of this material for the implementation in dye-sensitized solar cell photoanodes.
\end{abstract}

DOI: $10.12693 /$ APhysPolA.123.386

PACS: 81.15.Cd, 68.55.A-, 68.55.J-, 81.05.Rm, 72.20.Jv, 72.40.+w, 73.40.Mr, 88.40.H-

\section{Introduction}

Zinc oxide is well known as a promising alternative to titanium dioxide for the fabrication of photoanodes in dye-sensitized solar cells (DSCs) [1]. $\mathrm{ZnO}$ is a wide band gap semiconductor (energy gap $3.37 \mathrm{eV}$ ), with a conduction band edge positioned approximately at the same level as in $\mathrm{TiO}_{2}$, meaning that ruthenium-based dyes developed for $\mathrm{TiO}_{2}$ can efficiently work also when linked to $\mathrm{ZnO}$. With respect to titania, $\mathrm{ZnO}$ presents higher electron mobility and carrier lifetime, being these parameters very important to improve the performances of DSCs. Moreover, zinc oxide is well known for its ability to easily grow in a wide variety of nanostructures, like nanoparticles [2], nanowires or nanorods [3], nanotubes [4], nanosheets [5], nanoplants [6] and nanotetrapods [7], just to cite some valuable examples. The ability to tune the morphology at the nanoscale can open wide opportunities in the control of charge transfer dynamics in the photoelectrode. In fact, by opportunely tuning the morphology, it is possible to combine the fast direct transport in 1D or quasi 1D nanostructures (like nanowires and nanorods) with the wide exposed area for dye-sensitization typical of mesoporous layers.

We report here on the fabrication and characterization of dye-sensitized solar cells with $\mathrm{ZnO}$-based photoanodes with valuable efficiency. A simple, low cost and scalable method for the deposition of sponge-like mesoporous $\mathrm{ZnO}$

\footnotetext{
* corresponding author; e-mail: stefano.bianco@iit.it
}

nanostructures was used. A nanostructured $\mathrm{Zn}$ film is grown on glass covered with fluorine-doped tin oxide by radio frequency (rf) magnetron sputtering technique and subsequently subjected to a thermal treatment in oxygen atmosphere. The structural characterization of the material shows the formation of a pure wurtzite crystalline structure, without metallic inclusions. A coral-like structured film is formed, showing the superimposition of small branches, with an almost isotropic growth in length. The size and spacing of the nanostructures are on the order of the exciton diffusion length, ensuring optimal electron collection efficiency. The optimization of the sensitization procedure using Ru-based dye was performed, and a photovoltaic conversion efficiency as high as $4.83 \%$ under AM1.5G illumination was demonstrated.

\section{Experimental details}

\subsection{Materials and methods}

Fluorine-doped tin oxide (FTO) covered glasses $(7 \Omega /$ sq, Solaronix) were used as substrates. They were cleaned with "piranha" solution (sulfuric acid and hydrogen peroxide in 3:1 volume ratio) and covered with a hard mask. The nanostructured $\mathrm{Zn}$ film was grown by rf magnetron sputtering starting from a pure (99.99\%) $\mathrm{Zn}$ target, using Ar plasma. The chamber was evacuated down to a pressure of about $10^{-7}$ Torr, and no intentional heating was applied to the substrates. The $\mathrm{Zn}$ film was subsequently thermally oxidized at $380^{\circ} \mathrm{C}$ for $60 \mathrm{~min}$ in ambient condition. Details of the growth procedure are reported elsewhere [8]. 
Photoelectrodes were heated at $70{ }^{\circ} \mathrm{C}$ and soaked into a $0.25 \mathrm{mM}$ N719 dye solution (Ruthenizer535bis-TBA, Solaronix) in ethanol for different times at room temperature and then rinsed in ethanol to remove the un-adsorbed dye molecules. The counterelectrode was constituted by a glass/FTO sheet with a thin $(5 \mathrm{~nm})$ layer of Pt deposited on it by thermal evaporation.

DSCs were assembled using a microfluidic architecture $[9,10]$. A liquid $I^{-} / I_{3}^{-}$electrolyte (Iodolyte AN50, Solaronix) was used. All cells had an active area of $0.78 \mathrm{~cm}^{2}$ and measurements were performed with a $0.22 \mathrm{~cm}^{2}$ black rigid mask.

\subsection{Characterizations}

The morphology of the $\mathrm{Zn}$ and $\mathrm{ZnO}$ films was investigated by means of a ZEISS Supra 40 field emission scanning electron microscope (FESEM). The topography was investigated both in top view and in cross view (electron energy $5 \mathrm{keV}$ ).

$\mathrm{X}$-ray diffraction technique was used to determine the crystalline structure of the films (Panalytical PW1140-PW3020, $\mathrm{Cu} K_{\alpha}$ X-ray source). The scans were performed in a parallel beam geometry with a fixed angle of incidence $\omega=1.5^{\circ}$, in order to minimize the contribution of the substrate to the observed diffracted intensities.

The Brunauer-Emmett-Teller (BET) specific surface area of the $\mathrm{ZnO}$ sample was evaluated from $\mathrm{N}_{2}$ sorption isotherms (Quantachrome Autosorb1) by multipoint method within the relative pressure range of $0.1-$ $0.3 P / P_{0}$.

$I-V$ electrical characterizations under AM1.5G illumination $\left(1000 \mathrm{~W} / \mathrm{m}^{2}\right)$ were carried out using a class $\mathrm{A}$ solar simulator (91195A, Newport) and a Keithley 2440 source measure unit. Electrochemical impedance spectra were collected under the same illumination using an electrochemical workstation (760D, $\mathrm{CH}$ Instruments) in the frequency range $10^{-1}-10^{5} \mathrm{~Hz}$, at open circuit voltage. The amplitude of the AC signal was $10 \mathrm{mV}$.

\section{Results and discussion}

In Fig. 1a the morphology of the deposited film as evaluated by FESEM is presented. The $\mathrm{Zn}$ film shows a sponge-like structure, compatible with the results proposed by Baker et al. for $\mathrm{Zn}-\mathrm{Al}$ coatings [11]. The nanostructuring of a metallic film in a sponge-like structure occurs for growth processes performed with a substrate temperature $T$ approximately equal to a half of the melting temperature $T_{\mathrm{m}}$ (e.g. $\left.T / T_{\mathrm{m}} \approx 0.5\right)$ [12]. Being the melting temperature for zinc particularly low, it is possible to synthesize a porous nanostructured layer for $T \sim T_{\mathrm{amb}}$. Films with thicknesses up to $15 \mu \mathrm{m}$ were deposited.

As depicted in Fig. 1b, no significant morphological variation can be noticed after the high temperature oxidation procedure, resulting only in a moderate volume expansion. Top view in Fig. 1c allows appreciating the porous morphology of the film and its similarity with the

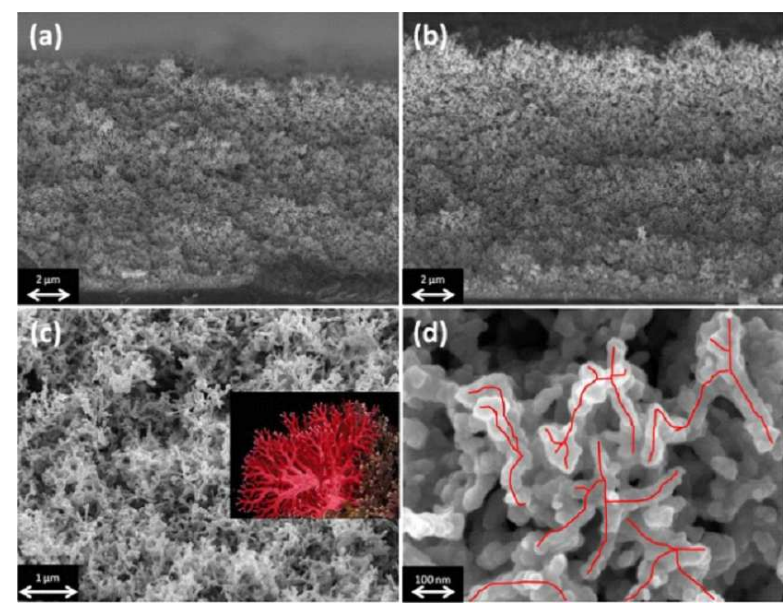

Fig. 1. FESEM characterization: (a) Zn film, cross view; (b) $\mathrm{ZnO}$ film, cross view; (c) $\mathrm{ZnO}$ film, top view, and (inset) visual comparison with a natural coral; (d) magnified image of the coral-like morphology, where the branched structure is evidenced.

structure of natural coral. The specific exposed surface, as measured by BET, was equal to $14.1 \mathrm{~m}^{2} / \mathrm{g}$, in line with what was measured for mesoporous $\mathrm{ZnO}$ layers deposited with other techniques [2]. In the magnified FESEM picture in Fig. 1d it is possible to observe the 3-dimensional coral-like nanostructure, formed by the superimposition of small branches able to grow in length along basically every direction. The typical dimension of the particulate is around $40 \mathrm{~nm}$, with spacing between adjacent structures in the range $10-60 \mathrm{~nm}$. Taking into account that the typical exciton length in $\mathrm{ZnO}$ is in the range $5-20 \mathrm{~nm}$, such morphological feature presents all the desired characteristics for efficient dye loading and charge transport towards the electrode [13].

The macroscopic appearance of the deposited film before and after the oxidation treatment drastically changes, moving from a black to a transparent feature, as shown in Fig. 2a. In Fig. 2b, the XRD characterization of the film before and after the oxidation procedure is reported. The diffraction pattern after the thermal treatment witnesses a complete oxidation of the film, without the formation of $\mathrm{Zn}$ residuals, which could be detrimental for charge transport in DSC photoelectrodes.

Photovoltaic characterizations were performed using a microfluidic architecture, which is an advantageous tool for the optimization of the process, being the assembly procedure simple and fast in a controlled and reproducible way [14]. A picture of the final device is shown in Fig. 2c. The sensitization procedure is known to be critical for $\mathrm{ZnO}$ electrodes [15], since it is necessary to avoid the partial $\mathrm{ZnO}$ dissolution in the dye solution. In Fig. 3 the $I-V$ curves and the evaluation of photovoltaic conversion efficiency (PCE) as a function of dye incubation time are reported. PCE was evaluated starting from the $I-V$ curves as 
(a)
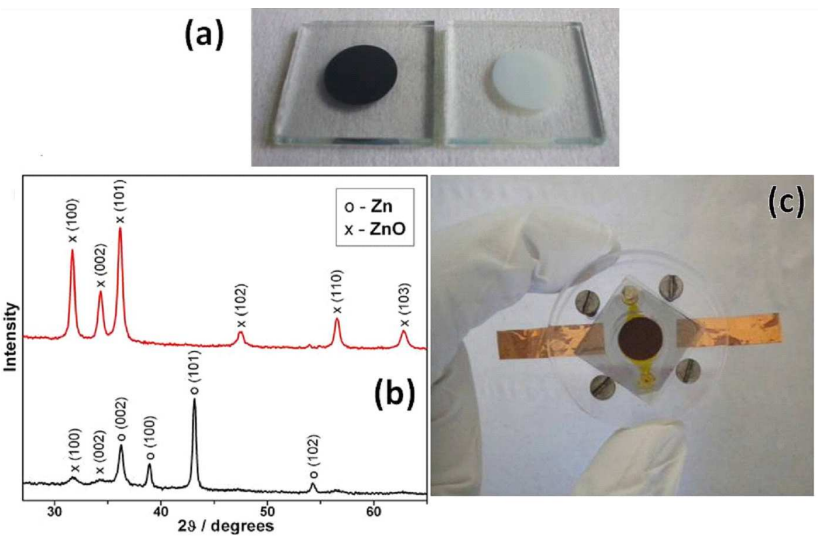

Fig. 2. (a) Picture of the photoanode, before (left) and after (right) the oxidation treatment; (b) XRD characterization, before and after the oxidation treatment (JCPDS cards: Zn 87-0713, ZnO 89-1397); (c) picture of the microfluidic cell.

$$
\mathrm{PCE}=\frac{F F V_{\mathrm{oc}} J_{\mathrm{sc}}}{P_{\mathrm{in}}}
$$

$V_{\mathrm{oc}}$ is the open circuit voltage, $J_{\mathrm{sc}}$ is the short circuit current density, $P_{\text {in }}$ is the input power density, and $F F$ is the fill factor, defined as $\left(V_{\max } J_{\max }\right) /\left(V_{\mathrm{oc}} J_{\mathrm{sc}}\right)$, where $V_{\max }$ and $J_{\max }$ are the voltage and current density at maximum power output, respectively.

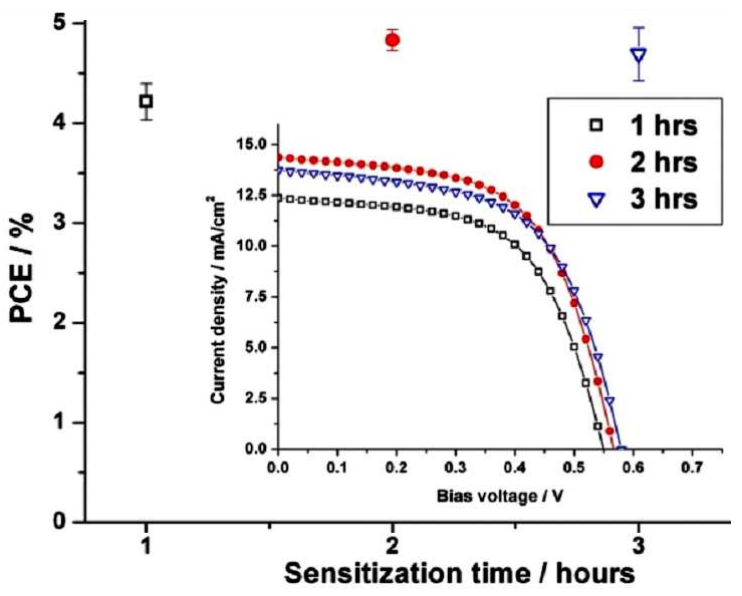

Fig. 3. Evaluation of the photovoltaic conversion efficiency in microfluidic cells for different sensitization times, starting from $I-V$ curves (inset). The thickness of the $\mathrm{ZnO}$ film is $15 \mu \mathrm{m}$.

Photoanodes with a $\mathrm{ZnO}$ film thickness equal to $15 \mu \mathrm{m}$ were used. Several devices were tested and the relative error between different samples was within the $5 \%$, meaning a very high reproducibility. An incubation time of two hours was found to be optimal for the sensitization of this material, in line with previously reported data [10] and for materials synthesized with other techniques [16]. In particular, an evident increase in $J_{\mathrm{sc}}$ is noticed, while $F F$ is almost independent of the sensitization procedure.
The efficiency increase for short incubation times is related with the slow kinetic of dye adsorption on the surface of the semiconductor. When the number of chemisorbed dye molecules on the surface of $\mathrm{ZnO}$ is low, the PCE is reduced both because of the small number of photogenerated charges injected in the semiconductor and because of the higher recombination rate with the electrolyte, occurring at the semiconductor unoccupied sites. When the optimal incubation time is exceeded, the formation of molecular aggregates between dissolved $\mathrm{Zn}^{2+}$ ions and dye molecules occurs [17]. The aggregates are inactive in terms of charge generation and act as filters for the impinging light, decreasing the overall efficiency of the device.

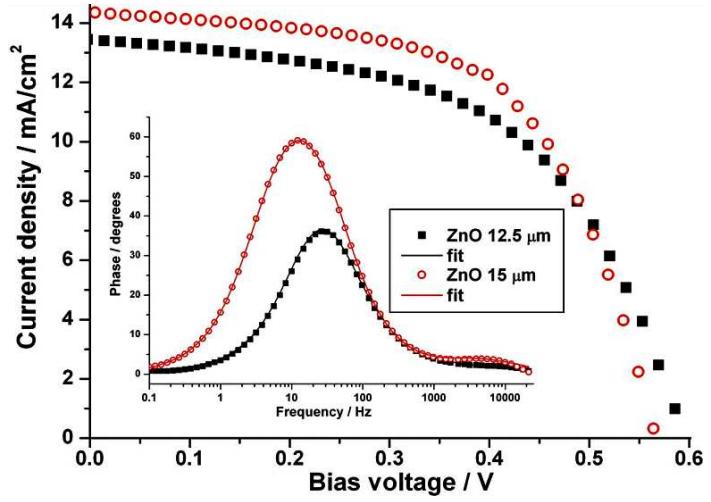

Fig. 4. $I-V$ and EIS (Bode plot, inset) characterization of $\mathrm{ZnO}$-based DSCs fabricated with different photoanode thicknesses, $12.5 \mu \mathrm{m}$ (black) and $15 \mu \mathrm{m}$ (red) The sensitization time was $2 \mathrm{~h}$.

With the optimized sensitization time of two hours, the photovoltaic behavior of the device as a function of the $\mathrm{ZnO}$ film thickness was tested. The results of the $I-V$ characterization and the Bode representation of the electrochemical impedance spectroscopy (EIS) phase are reported in Fig. 4. The increase in photoanode thickness from $12.5 \mu \mathrm{m}$ to $15 \mu \mathrm{m}$ in the microfluidic architecture allowed obtaining a slightly higher efficiency, with an increase on the short circuit current, while the $F F$ value remained unaffected.

Thicker photoelectrodes are able to load a higher amount of dye, thus allowing the injection of a higher number of carriers in the conduction band of the semiconductor, and the charge transport is efficient enough to allow the collection of the electrons at the FTO electrode. More interestingly, the carrier lifetime shows a dependence on the photoanode thickness. The experimental curves of EIS were fitted using an equivalent circuit in order to obtain information about transport and recombination of charges [18]. In particular, the electron lifetimes at open circuit voltage can be estimated from the frequency $f$ giving the maximum in the Bode plot reported in Fig. 4 b, as $\tau=1 /(2 \pi f)$. The evaluated charge carrier lifetimes at $V_{\text {oc }}$ were equal to $12 \mathrm{~ms}$ and $48 \mathrm{~ms}$ for the $12.5 \mu \mathrm{m}$ and $15 \mu \mathrm{m}$ thick $\mathrm{ZnO}$ photoan- 
odes, respectively. The dependence of electron lifetime on the photoanode thickness is probably related with the contribution of the back transfer of electrons at the FTO-electrolyte interface [19]. For thicker photoanodes, this parasitic effect loses importance, giving as a macroscopic result a higher overall carrier lifetime, as reported previously for mesoporous $\mathrm{TiO}_{2}$ films [20].

\section{Conclusions}

The fabrication and characterization of DSCs made with a coral-shaped $\mathrm{ZnO}$ photoanodes is reported. A simple and scalable method for the synthesis of sponge-like $\mathrm{Zn}$ structure was presented. The $\mathrm{Zn}$ nanostructures were oxidized with a simple thermal treatment in air, obtaining a porous $\mathrm{ZnO}$ film with a $3 \mathrm{D}$ coral-like morphology. XRD characterization showed the complete oxidation of the film.

The solar energy conversion efficiency of DSCs based on $\mathrm{ZnO}$ films sensitized with Ru-based dyes was evaluated. The sensitization procedure was refined, being the optimal sensitization time equal to $2 \mathrm{~h}$. Using a $15 \mu \mathrm{m}$ thick photoanode assembled in a microfluidic architecture, a noticeable photovoltaic conversion efficiency of $4.83 \%$ was evaluated, with a charge carrier lifetime at open circuit voltage equal to $48 \mathrm{~ms}$.

\section{Acknowledgments}

Authors would like to thank Dr. D. Manfredi for FESEM characterizations and Dr. M. Armandi for BET measurement.

\section{References}

[1] Q. Zhang, C.S. Dandeneau, X. Zhou, G. Cao, Adv. Mater. 21, 4087 (2009)

[2] K. Keis, E. Magnusson, H. Lindstrom, S.E. Lindquist, A. Hagfeldt, Sol. Energy Mater. Sol. Cells 73, 51 (2002)

[3] D. Calestani, M.Z. Zha, L. Zanotti, M. Villani, A. Zappettini, Cryst. Eng. Comm. 13, 1707 (2011).

[4] Q.C. Li, V. Kumar, Y. Li, H.T. Zhang, T.J. Marks, R.P.H. Chang, Chem. Mater. 17, 1001 (2005)
[5] J.H. Xiang, P.X. Zhu, Y. Masuda, M. Okuya, S. Kaneko, K. Koumoto, J. Nanosci. Nanotechnol. 6, 1797 (2006)

[6] A. Tiwari, M. Snure, J. Nanosci. Nanotechnol. 8, 3981 (2008)

[7] D. Calestani, M. Zha, R. Mosca, A. Zappettini, M.C. Carotta, V. Di Natale, L. Zanotti, Sens. Actuat. B 144, 472 (2010)

[8] R. Gazia, A. Chiodoni, S. Bianco, A. Lamberti, M. Quaglio, A. Sacco, E. Tresso, P. Mandracci, C.F. Pirri, Thin Solid Films 524, 107 (2012)

[9] A. Lamberti, A. Sacco, S. Bianco, E. Giuri, M. Quaglio, A. Chiodoni, E. Tresso, Microelectron. Eng. 88, 2308 (2011)

[10] A. Lamberti, R. Gazia, A. Sacco, S. Bianco, M. Quaglio, A. Chiodoni, E. Tresso, C.F. Pirri, "Coral-shaped $\mathrm{ZnO}$ nanostructures for dye-sensitized Solar Cell photoanodes", Progr. Photovolt. Res. Appl. in press, 2012.

[11] M.A. Baker, W. Gissler, S. Klose, M. Trampert, F. Weber, Surf. Coat. Technol. 125, 207 (2000).

[12] A.F. Jankowski, J.P. Hayes, J. Vac. Sci. Technol. A 21, 422 (2003)

[13] I. Gonzalez-Valls, M. Lira-Cantu, Energy Environ. Sci. 2, 19 (2009)

[14] A. Sacco, A. Lamberti, M. Quaglio, S. Bianco, E. Tresso, A.-L. Alexe-Ionescu, C.F. Pirri, Int. J. Photoenergy 2012, 216780 (2012).

[15] T.P. Chou, Q. Zhang, G. Cao, J. Phys. Chem. C 111, 18804 (2007)

[16] N. Memarian, I. Concina, A. Braga, S.M. Rozati, A. Vomiero, G. Sberveglieri, Angew. Chem. Int. Ed. 123, 12321 (2011)

[17] K. Keis, J. Lindgren, S.E. Lindquist, A. Hagfeldt, Langmuir 16, 4688 (2000).

[18] Y. Liu, X. Sun, Q. Tai, H. Hu, B. Chen, N. Huang, B. Sebo, X.-Z. Zhao, J. Power Sources 196, 475 (2011).

[19] P.J. Cameron, L.M. Peter, J. Phys. Chem. B 109, 7392 (2005)

[20] S.M. Waita, B.O. Aduda, J.M. Mwabora, C.G. Granqvist, S.-E. Lindquist, G.A. Niklasson, A. Hagfeldt, G. Boschloo, J. Electroanal. Chem. 605, $151(2007)$ 